Un théorème limite sur la dérivée tangentielle du potentiel généralisé de simple couche relatif au système parabolique.

par W. Żakowski (a Varsovie, Pologne)

Résumé. * L'auteur a démontré l'existence de la limite de la dérivée tangentielle $D_{s_{p}}\left[D_{X}^{M-\dot{2}} V_{\alpha}(X, t)\right],(x=1, \ldots, N)$, du potentiel généralisé de simple conche (7) relatif au systène parabolique (1), si $X \rightarrow P \in S$.

1. Introduction et les formules fondamentales. - Soit le système para* bolique de $N \geq 1$ équations aux dérivées partielles d'ordre $M \geq 2$ de la forme

$$
\begin{gathered}
\widehat{\Psi}^{(\alpha)}\left(u_{1}, \ldots, u_{N}\right) \equiv \sum_{1 \leq j \leq N}^{0 \leq k_{1}+\ldots+k_{n} \leq M} A_{\alpha j}^{k_{1} \ldots k_{n}}(X, t) \frac{\partial^{k_{1}+\ldots+k_{n}} \dot{u}_{j}}{\partial x_{1}^{k_{1}} \ldots \partial x_{n}^{k_{n}}}-\frac{\partial u_{\alpha}}{\partial t}=0 \\
(\alpha=1,2, \ldots, N)
\end{gathered}
$$

aux fonctions inconnues $u_{1}(X, t), \ldots, u_{N}(X, t)$. Les coefficients $A_{\alpha j}^{k_{3} \ldots k_{n}}(X, t)$ sont des fonctions complexes, définies, continues et bornées dans la région

$$
X\left(x_{1}, \ldots, x_{n}\right) \in E^{n} ; \quad 0 \leq t \leq T .
$$

$E^{n}$ désigne un espace euclidien à $n$ dimensions, $n \geq 2$.

Nous admettons les hypothèses suivantes.

I. Conformément à la définition du système parabolique, donnée par I. Petrovskx [1], nous admettons que l'équation

$$
\underset{\alpha, j}{\operatorname{det}}\left|\underset{k_{1}+\ldots+k_{n}=M}{\Sigma} A_{\alpha j}^{k_{1} \ldots k_{n}}(X, t)\left(i s_{1}\right)^{k_{1} \ldots} \ldots\left(i s_{n}\right)^{k_{n}}-\delta_{\alpha}^{j} \lambda\right|=0
$$

admet les racines en $\lambda$, dont toutes les parties réelles sont inférieures où égales à un nombre négatif fixé - $\delta$

$$
\operatorname{Re}(\lambda)<-\delta<0
$$

pour toutes les valeurs des variables réelles $s_{1}, \ldots, s_{n}$ vérifiant l'égalité $s_{i}^{2}+\ldots+s_{n}^{2}=1$ et pour tout point de la région (2).

II. Les coefficients $A_{\alpha j}^{k_{1} \ldots k_{n}}(X, t)$ sont hölderiens par rapport à la variable spatiäle $X$ à l'exposant $h$, uniformément continus par rapport à la 
variable $t$ et en outre les coefficients des dérivées de l'ordre le plus élevé $M$ sont hölderiens par rapport à la variable $t$, à l'exposant $h^{\prime}$.

Dans le travail [2] W. Pogonzelski a construit pour le système (1) une matrice de solutions, $\left\{\Gamma_{\alpha \beta}(X, t ; Y, \tau)\right\}, \alpha, \beta=1,2, \ldots, N$, nommée par lui "matrice des solutions fondamentales》 en admettant que les coefficients $A_{\alpha j}^{k_{1}, \ldots k_{n}}(X, t)$ vérifient les hypothèses I et II. Les èléments $\Gamma_{\alpha \beta}(X, t ; Y, \tau)$ dè cette matrice sont donnés par la formule

$$
\mathrm{I}_{\alpha \beta}(X, t ; Y, \tau)=W_{\alpha \beta}^{X, \tau}(X, t ; Y, \tau)+\bar{W}_{\alpha \beta}(X, t ; Y, \tau)
$$

où $X\left(x_{1}, \ldots, x_{n}\right)$ et $Y\left(\xi_{1}, \ldots, \xi_{n}\right)$ sont les deux points différents, arbitraires, de l'espace $E^{n}$, $\tau<t$ deux valeurs arbitraires dans l'intervalle $(0, T)$, $W_{\alpha \beta}^{Y, \tau}(X, t ; Y, \tau)$ sont les éléments de la matrice des quasi-solutions du système (1), et

$$
\bar{W}_{\alpha \beta}(X, t ; Y, \tau)=\int_{\tau}^{t} \iint_{E(\Pi)} \int_{\sum^{\prime}} \sum_{\gamma=1}^{N} W_{\alpha_{\gamma}}^{\Pi, \zeta}(X, t ; \Pi, \zeta) \Phi_{\alpha \beta}(\Pi, \zeta ; Y ; \tau) d \Pi d \zeta
$$

où les fonctions $\Phi_{1 \beta}, \ldots, \Phi_{N \beta}$ des couples $\Pi$, $\zeta$ et $Y$, $\tau$ forment pour chaque $\beta$ fixé une solution du système d'équations intégrales de Voltenna [2]; $Y, \tau$ jouent un rôle des paramètres fixés.

Soit l'intégrale de surface

$$
V(X, t)=\int_{0}^{t} \iint_{S(Q)} \sum_{\beta=1}^{N} \Gamma_{\alpha \beta}(X, t ; Q, \tau) \varphi_{\beta}(Q, \tau) d Q d \tau, \quad(\alpha=1,2, \ldots, N)
$$

dite potentiel généralisé de simple couche, relatif au système parabolique (1'). Nous admettons encore les deux hypothèses suivantes.

III. La surface $S$ à $n-1$ dimensions (qui en général n'est pas fermée) vérifie les conditions connues de Liapounofr dont l'une, concernant l'angle $\Delta(P, Q)$ entre les normales aux points $P$ et $Q$ de la surface $S$, a la forme

$$
\Delta(P, Q)<\text { const }|P Q|^{h_{L},} \quad\left(0<h_{L} \leq 1\right) .
$$

IV. Les $N$ fonctions de la suite $\left\{\varphi_{\beta}(Q, t\}\right.$, dite densité de la simple couche, sont définies dans la région $Q \in S, 0 \leq t \leq T$, continues par rapport à la variable $t$ pour $0 \leq t \leq T$ et vérifient la condition de HöLDER relative. ment aux points $Q$ de la surface $S$, à l'exposant $h_{\varphi}$

$$
\left|\varphi_{\beta}(Q, \tau)-\varphi_{\beta}\left(Q_{1}, \tau\right)\right|<\mathrm{const}\left|Q Q_{1}\right|^{h_{\varphi}} \quad(\beta=1,2, \ldots, N) .
$$


Les fonctions (7) ont les dërivées spatiales d'ordre $m=1,2, \ldots, M$ déterminées par les intégrales regulières

$$
D_{X}^{m}\left[V_{\alpha}(X, t)\right]=\int_{0}^{t} \int_{S(Q)} \int_{\beta=1}^{N} D_{X}^{m}\left[\Gamma_{\alpha \beta}(X, t ; Q, \tau)\right] \mathscr{Y}_{\beta}(Q, \tau) d Q d \tau
$$

en tout point $X \in E^{n}-S$ pour $0<t<T$.

On sait que les derivées d'ordre $m=1,2, \ldots, M-2$ des éléments de la matrice des solutions vérifient l'inégalité

$$
\left|D_{X}^{m}\left[\Gamma_{\alpha \beta}(X, t ; Q, \tau)\right]\right|<\frac{\text { const }}{(t-\tau)^{\mu}} \cdot \frac{1}{|X Q|^{n+m-M_{\mu}}}
$$

qui contient les fonotions aux singularites faibles si nous choisivons la gonstante $\mu$ dans l'intervalle

$$
\frac{m+1}{M}<\mu<\min \left(1, \frac{m+n}{M}\right)
$$

puisque on a $n+m-M \mu<n-1$, si $m=0,1,2, \ldots, M-2$. Nous en concluons l'existence des valeurs limites

$$
\lim _{X \rightarrow P \in S} D_{X}^{m}\left[V_{\alpha}(X, t)\right]=\int_{0}^{t} \int_{S(Q)} \int_{\beta=1}^{N} D_{X}^{m}\left[\Gamma_{\alpha \beta}(P, t ; Q, \tau)\right] \varphi_{\beta}(Q, \tau) d Q d \tau
$$

pour $m=0,1, \ldots, M-2$, les fonctions $\varphi_{\beta}(Q)$, $)$ n'étant qu'intégrables. Ce raisonnement ne concerne pas les dérivées d'ordre $m=M-1$.

Dans ce travail nous démontrerons sous l'hypothèses I-IV l'existence de la limite de la dérivée de l'intégrale

$$
D_{X}^{M-2}\left[V_{\alpha}(X, t)\right]=\int_{0}^{t} \int_{S(Q)} \int_{\beta=1}^{N} D_{X}^{M-2}\left[\Gamma_{\alpha \beta}(X, t ; Q, \tau)\right] \varphi_{\beta}(Q, \tau) d Q d \tau
$$

dans la direction d'une tangente au point arbitraire $P \in S$ (non situe sur le bord de cette surface), si $X \rightarrow P$, par une méthode analogue que dans le travail [3].

2. Existence de la limite de la iérivée tangentielle. - D'après la formule (5), nous pouvons ecrire l'intégrale (7) sous la forme d'une somme des 
intégrales

$$
V_{\alpha}(X, t)=U_{\alpha}(X, t)+\bar{U}_{\alpha}(X, t)
$$

où

$$
U_{\alpha}(X, t)=\int_{0}^{t} \int_{S(Q)} \int_{\beta=1}^{N} W_{\alpha \dot{\beta}}^{O_{\beta}}(X, t ; Q, \tau) \varphi_{\beta}(Q, \tau) d Q d \tau
$$

et

$$
\begin{gathered}
\bar{U}_{\alpha}(X, t)=\int_{0}^{t} \int_{S(Q)} \int_{\beta=1}^{N} \bar{W}_{\alpha \beta}(X, t ; Q, \tau) \varphi_{\beta}(Q, \tau) d Q d \tau \\
(\alpha=1,2, \ldots, N) .
\end{gathered}
$$

D'après le travail [2], les dérivées spatiales d'ordre $M-1$ des fonctions (6) admettent les limitations aux singularités separées suivantes

$$
\left|D_{X}^{M-1}\left[\bar{W}_{\alpha \beta}(X, t ; Q, \tau)\right]\right|<\frac{1}{(t-\tau)^{\mu_{0}}} \cdot \frac{\text { const }}{|X Q|^{n-1+M\left(1-p_{0}\right)-h_{1}}}
$$

où $h_{1}=\min \left(h, 2 h^{\prime}\right), \mu_{0}$ est un nombre positif arbitraire dans l'intervalle ouvert $\left(1-\frac{1+h_{1}}{M}, 1\right)$; par conséquent nous aurons

$$
n-1+M\left(1-\mu_{0}\right)-h_{1}<n-1
$$

si $\mu_{0}$ est choisi dans l'intervalle ouvert $\left(1-\frac{h_{1}}{M}, 1\right)$ nous pouvons done affirmer que la dérivée

$$
D_{X}^{M-1} \bar{U}_{\alpha}(X, t)=\int_{0}^{t} \int_{S(Q)} \int_{\beta=1} \sum_{\beta=1}^{N} D_{X}^{M-1}\left[\bar{W}_{\alpha \beta}(X, t ; Q, \tau)\right] \varphi_{\beta}(Q, \tau) d Q d \tau
$$

tend uniformement vers la limite determinee par l'integrale absolument convergent

$$
\lim _{X \rightarrow P} D_{X}^{M-1}\left[\bar{U}_{\alpha}(X, t)\right]=\int_{0}^{t} \iint_{S(Q)} \sum_{\beta=1}^{N} D_{X}^{M-1}\left[\bar{W}_{\alpha \beta}(P, t ; Q, \tau)\right] \varphi_{\beta}(Q, \tau) d Q d \tau
$$

si le point $X \in E^{n}-S$ tend d'une facon arbitraire vers le point arbitraire $P \in S$ (non situé sur le bord de cette surface). 
Les dérivées spatiales d'ordre $M-1$ de quasi-solutions admettent les limitations aux singularités séparées suivantes:

$$
\left|D_{X}^{M-1} W_{\alpha \beta}^{Q, \tau}(X, t ; Q, \tau)\right|<\frac{1}{(t-\tau)^{\mu}} \cdot \frac{\text { const }}{|X Q|^{n-1+M(1-\mu)}}
$$

où $\mu$ est un nombre positif arbitraire inférieur à l'unité; par conséquent

$$
n-1+M(1-\mu)>n-1
$$

quelque soit le choix de la constante $\mu$ dans l'intervalle ouvert $(0,1)$ et étude de l'existence de la limite de la dérivée de l'integrale (10) dans la direction d'une tangente à la surface $S$ exige des considérations plus délicates.

Nous allons démontrer le théorème suivant.

Théonème. - Si les hypothèses I, II, III et IV (roir introduction) sont satisfaites, alors la dérivée de l'intégrale $(10)$ an point $X \in E^{n}-S$ dans la direction d'une tangente arbitraire $s_{P}$ a la surface $S$ au point arbitraire $P \in S$ (non situé sur le bord de cette surface) tend vers une limite déterminée par l'intégrale singulière itérée

$$
D_{s_{P}}\left[D_{X}^{M-2} V_{\alpha}(X, t)\right] \underset{X \rightarrow P}{\longrightarrow} \int_{0}^{t} \int_{S(Q)} \int_{\beta=1} \sum_{\beta=1}^{N} D_{s_{p}}\left[D_{X}^{M-2} \Gamma_{\alpha \beta}(P, t ; Q, \tau)\right] \varphi_{\beta}(Q, \tau) d Q d \tau
$$

(qui en général n'est pas absolument convergente) si le point $X$ tend vers le point $P \in S$ le long de la normale en $P$.

Dhmonstration. - Supposons que le point $X \in E^{n}-S$ tend vers le point intérieur $P \in S$ le long de la normale en $P$ et considérons un système d'axes rectangulaires $\left(P x_{1}, P x_{2}, \ldots, P x_{n}\right)$ où l'axe $P x_{n}$ est normal à la surface $S$; done les axes $P x_{1}, P x_{2}, \ldots, P x_{n-1}$ sont situés dans le plan tangent en $P$ à la surface $S$, nous admettons que l'axe $P x_{1}$ coüncide avec la direction $s_{P}$.

D'après l'existence de la limite (20) il suffit d'étudier la dérivée

$$
\begin{gathered}
D_{x_{1}}\left[D_{X}^{M-2} U_{\alpha}(X, t)\right]=\int_{0}^{t} \int_{S(Q)} \int_{\beta=1}^{N} D_{\varkappa_{1}}\left[D_{X}^{M-2} W_{\alpha \beta}^{Q, \tau}(X, t ; Q, \tau)\right] \rho_{\beta}(Q, \tau) d Q d \tau \\
(\alpha=1,2, \ldots, N),
\end{gathered}
$$

si $X \rightarrow P$.

Soit un eylindre $W$, ayant pour l'axe $P x_{n}$, de rayon $\delta_{0} q u i$ découpe de la surface $S$ au voisinage du point $P$ une portion $S_{W}$ et du plan tangent en $P$ une hypersphère $S_{W}^{\prime}$ à $n-2$ dimensions-projection de la portion $S_{W}$. 
D'après les conditions de LiaPounofF, (hypothèse III), nous pouvons toujours fixer le rayon $\delta_{0}$ suffisamment petit, pour que les points de la projection $S^{\prime}{ }_{W}$ correspondaient d' une façon biunivoque au points de la portion $S_{W}$.

Nous décomposons l'intégrale (24) en deux parties

$$
D_{x_{1}}\left[D_{X}^{M-2} U_{\alpha}(X, t)\right]=D_{x_{1}}\left[D_{X}^{M-2} U_{\alpha}(X, t)\right]_{S_{W}}+D_{x_{1}}\left[D_{X}^{M-2} U_{\alpha}(X, t)\right]_{S-S_{W}}
$$

étendues à la portion $S_{W}$ et à la surface extérieure $S-S_{W}$. Le second terme de la somme (25) est évidemment contina au point $P$ et nous aurons

$$
\lim _{X \rightarrow P} D_{x_{1}}\left[D_{X}^{M-2} U_{\alpha}(X, t)\right]_{S-S_{W}}=D_{x_{1}}\left[D_{X}^{M-2} U_{\alpha}(P, t)\right]_{S-S_{W}}=
$$

$$
=\int_{0}^{t} \iint_{S-S_{W}} \sum_{\beta=1}^{N} D_{x_{1}}\left[D_{X}^{M-2} W_{\alpha \beta}^{Q, \tau}(P, t ; Q, \tau)\right] \cdot \varphi_{\beta}(Q, \tau) d Q d \tau
$$

Pour étudier la prèmiere des intégrales (25) nous l'écrivons de la façon suivante

$D_{\alpha 1}\left[D_{X}^{M-2} U_{\alpha}(X, t)\right]_{s_{W}}=\int_{0}^{t} \int_{S_{W}} \int_{\beta=1}^{N} D_{x_{1}}^{N}\left[D_{X}^{M-2} W_{\alpha \beta}^{Q, \tau}(X, t ; Q, \tau]\left[\varphi_{\beta}(Q, \tau)-\varphi \beta(P, \tau)\right] d Q d \tau+\right.$

$$
+\int_{0}^{t} \sum_{\beta=1}^{N} \varphi_{\beta}(P, \tau) \iint_{S_{W}} D_{\varkappa_{1}}\left[D_{X}^{M-2} W_{\alpha \beta}^{Q, \tau}(X, t ; Q, \tau)\right] d Q d \tau=I_{1}^{(\alpha)}(X, t)+I_{2}^{(\alpha)}(X, t) .
$$

D'après l'inégalité de HöLDER pour la fonctions $\varphi_{\beta}(Q, \tau), \beta=1,2, \ldots, N$, (hypothèse IV) et l'inégalité (21), le fonctions sous la signe de la première intégrale (27), vérifient l'inégalité

$$
\begin{gathered}
\mid \sum_{\beta=1}^{N} D_{x_{1}}\left[D_{X}^{M-2} W_{\alpha \beta}^{Q, \tau}(X, t ; Q, \tau)\right]\left[\varphi_{\beta}(Q, \tau)-\psi_{\beta}(P, \tau] \mid<\frac{\text { const }}{(t-\tau)^{\mu}} \cdot \frac{|P Q|^{h_{\phi}}}{\left.|X Q|^{n-1+M(1-\mu}\right)}\right. \\
(\alpha=1,2, \ldots, N)
\end{gathered}
$$

Done, en fixant l'exposant $\mu$ à l'intérieur de l'intervalle $\left(1-\frac{h_{\varphi}}{M}, 1\right)$ 
nous pouvons affirmer, que $I_{1}^{\langle\alpha\rangle}(X, t)$ tend vers une limite

$$
\begin{gathered}
\lim _{X \rightarrow P} I_{1}^{(\alpha)}(X, t)=I_{1}^{(\alpha)}(P, t)= \\
=\int_{0}^{t} \int_{S_{W}} \int_{\beta=1} \sum_{x_{1}}^{N} D_{x_{1}}\left[D_{X}^{M-2} W_{\alpha \beta}^{Q, \tau}(P, t ; Q, \tau]\left[\varphi_{\beta}(Q, \tau)-\varphi_{\beta}(P, \tau)\right] d Q d \tau\right. \\
(\alpha=1,2, \ldots, N) .
\end{gathered}
$$

L'intégrale (29) est absolument convergente, puisque l'intégrale de sur. face, vérifie, (voir (28)), l'inégalité à faible singularité.

Pour étudier $I_{2}^{(\alpha)}(X, t)$, considérons l'intégrale auxiliaire

$$
I_{0}^{(\alpha)}(X, t)=\int_{0}^{t} \sum_{\beta=1}^{N} \varphi_{\beta}(P, \tau) \iint_{S_{W}^{\prime}} D_{x_{1}}\left[D_{X}^{M-2} W_{\alpha \beta}^{P, \tau}\left(X, t ; Q^{\prime}, \tau\right)\right] d Q^{\prime} d \tau
$$

étendue à la projection $S_{W}^{\prime}$ de la portion $S_{W}$ sur le plan tangent en $P$, $Q^{\prime}$-projection du point $Q$ sur ce plan. Les éléments de la matrice des quasi-solutions sont donnes par les integrales de Fodrien

$$
W_{\alpha \beta}^{P, \tau}\left(X, t ; Q^{\prime}, \tau\right)=\frac{1}{(2 \pi)^{n}} \int_{-\infty}^{+\infty} v_{\alpha}^{\beta}(t, \tau ; P, \tau ; S) \exp \left[i \sum_{\nu=1}^{n}\left(x_{y}-\xi_{y}\right) s_{\nu}\right] d S,
$$

$(\alpha, \beta=1,2, \ldots, N)$ étendues à tout l'espace enclidien $E^{n}$ des variables réelles $S\left(s_{1}, s_{2}, \ldots, s_{n}\right) ; \xi_{1}, \xi_{2}, \ldots, \xi_{n},\left(\xi_{n}=0\right)$, étant les coordonnées du point $Q^{\prime}$ et $x_{1}, x_{2}, \ldots, x_{n}-$ du point $X,\left(x_{1}=\ldots=x_{n-1}=0\right)$. Les fonctions $v_{1}^{\beta}, v_{2}^{\beta}, \ldots, v_{N}^{\beta}$, $(\beta=1,2, \ldots, N)$ pour $\beta$ fixé forment une solution du système d'équations différentielles ordinaires, $[2]$, où $P$, $\tau$ et $S$ jonent un rôle des parametres fixés.

D'après (31), nous aurons l'égalité des dérivées

$$
D_{x_{y}}\left[D_{X}^{M-2} W_{\alpha \beta}^{P, \tau}\left(X, t ; Q^{\prime}, \tau\right)\right]=-D_{\xi_{y}}\left[D_{X}^{M-2} W_{\alpha \beta}^{P, \tau}\left(X, t ; Q^{\prime}, \tau\right)\right]
$$

pour $\vee=1,2, \ldots, n$.

En appliquant la théorème de GreEr. nous pouvons donc écrire l'inté. grale (30) sous la forme d'une intégrale

$$
I_{0}^{(\alpha)}(X, t)=-\int_{0}^{t} \sum_{\beta=1}^{N} \varphi_{\beta}(P, \tau)\left\{\int_{C_{W}} D_{X}^{M-2}\left[W_{\alpha \beta}^{P, \tau}\left(X, t ; Q^{\prime}, \tau\right)\right] \cos \nu d l_{Q^{\prime}}\right\} d \tau
$$


étendue à la frontière $C_{W} \grave{a} \quad n-2$ dimensions du domaine $S_{W}^{\prime} ; \quad \vee$ désigne l'angle que fait la normale extérieure à la surface $C_{W}$ au point $Q^{\prime}$ avec l'axe $P_{x_{1}}$ et $d l_{Q^{\prime}}$ - l'élément d'aire de la surface $C_{W}$ au point $Q^{\prime}$.

Par conséquent, il est évident que la fonetion (33) tend vers une limite déterminée

(34) $\lim _{X \rightarrow P} I_{0}^{(\alpha)}(X, t)=-\int_{0}^{t} \sum_{\beta=1}^{N} \varphi_{\beta}(P, \tau)\left\{\int_{C_{W}} D_{X}^{M(-2}\left[W_{\alpha \beta}^{P, \tau}\left(P ; t ; Q^{\prime}, \tau\right)\right] \cos \vee d l_{Q^{\prime}}\right\} d \tau$

si $X$ tend vers $P$.

D'après (21), nous aurons

(35) $\left.D_{\alpha}^{M-2}\left[W_{\alpha \beta}^{P, \tau}\left(P, t ; Q^{\prime}, \tau\right)\right]=\frac{(i)^{M-2}}{(2 \pi)^{n}} \int_{-\infty}^{+\infty} v_{\alpha}^{\beta}(t, \tau ; P, \tau ; S)\left(s_{1}\right)^{p_{1}} \ldots\left(s_{n}\right)^{p_{n}} \cdot \exp \mid-i \sum_{\nu=1}^{n-1} s_{\nu} \xi_{y}\right] d S$

où $p_{1}, \ldots, p_{n}$ sont les nombres entiers non-négatifs et $p_{1}+p_{2}+\ldots+p_{n}=M-2$.

Les fonctions $v_{1}^{\beta}, v_{2}^{\beta}, \ldots, v_{N}^{\beta},(\beta=1,2, \ldots, N)$ pour $\beta$ fixé forment une solution du système d'équations différentielles ordinaires

$$
\frac{d v_{\alpha}^{\beta}}{d t}=\sum_{1 \leq j \leq N}^{k_{1}+\cdots+k_{n}=M} A_{\alpha j}^{k_{1} \ldots k_{n}}(Z, \zeta)\left(i s_{1}\right)^{k_{1}} \ldots\left(i s_{1}\right)^{k_{n}} v_{j}^{\beta}(t, \tau ; Z, \xi ; S)
$$

où $Z, \zeta, S, \tau$ jouent un rôle des paramètres fixés; ensuite, l'hypothèse I exige d'admettre que le degré $M$ des équations (1) est pair, done

$$
v_{\alpha}^{\beta}(t, \tau ; P, \tau ; S)=v_{\alpha}^{\beta}(t, \tau ; P, \tau ;-S)
$$

oủ $-S$ désigne la point $\left(-s_{1},-s_{2}, \ldots,-s_{n}\right)$. Par consequent, il est évident que l'intégrale

$$
\int_{C_{W}} D_{X}^{M-2}\left[W_{\alpha \beta}^{P, \tau}\left(P, t ; Q^{\prime}, \tau\right)\right] \cos v d l_{Q^{\prime}}
$$

est nulle d'après la symétrie centrale de la fonction

$$
D_{X}^{M-2}\left[W_{\alpha \beta}^{P, \tau}\left(P, t ; Q^{\prime}, \tau\right)\right]
$$

Nous aurons dono

$$
\lim _{X \rightarrow P} I_{0}^{(\alpha)}(X ; t)=I_{0}^{(\alpha)}(P, t)=0 .
$$


Il en résulte, d'après (30), (33), (34) et (40),

(41) $\left.\lim _{X \rightarrow P} I_{0}^{(\alpha)}(X, t)=\int_{0}^{t} \sum_{\beta=1}^{N} \varphi_{\beta} \mid P, \tau\right) \iint_{S_{W}^{\prime}} D_{\alpha^{\prime}}\left[D_{X}^{M-2} W_{\alpha \beta}^{P, \tau}\left(P, \tau ; Q^{\prime}, \tau\right)\right] d Q^{\prime} d \tau=0$

Nous signalons que cette convergence de l'intégrale n'est pas absolue, puisque l'intégrale itérée de la valeur absolue de la fonction $D_{X}^{M-1} W_{\alpha \beta}^{P_{\text {, }}}$ n'existe pas en général.

Considérons maintenant la différence entre les intégrales $I_{2}^{(\alpha)}(X, t)$ et $I_{\mathfrak{o}}^{(\alpha)}(X, t)$

$$
=\int_{0}^{t} \underbrace{N}_{\beta=1} \varphi_{\beta}\left(P, \tau \int_{S_{W}} \int_{W} D_{x_{1}}\left[D_{X}^{M-2} W_{\alpha \dot{\beta}}^{Q, \tau}(X, t ; Q, \tau)\right]-D_{x_{1} \mid} D_{X}^{M-2} W_{\alpha \dot{\beta}}^{P_{*} \tau}\left(X, t ; Q^{\prime}, \tau\right)\right] \gamma(Q)\} d Q d \tau
$$

$$
R^{(\alpha)}(X, t)=I_{2}^{(\alpha)}(X, t)-I_{0}^{(\alpha)}(X, t)=
$$

où $\gamma(Q)$ désigne la valeur absolue du cosinus d'angle que fait la normale à la surface $S$ au point $Q$ avec l'axe $P x_{n}$.

D'après les condition de Liapounoff (hypothèse III) nous aurons

$$
1-\gamma(Q) \leq \text { const }|P Q|^{2 h_{L},} \quad\left(0<h_{L} \leq 1\right)
$$

où $h_{L}$ jest l'exposant positif constant caractéristique pour la surface $S$.

Décomposons maintenant la différence sous le signe de l'intégrale de surface (42) de la façon suivante

$$
D_{X}^{M-1}\left[W_{\alpha, \beta}^{Q_{\beta} \tau}(X . t ; Q, \tau)\right]-D_{X}^{M-1}\left[W_{\alpha, \beta}^{P \cdot \tau}\left(X, t ; Q^{\prime}, \tau\right)\right] \gamma(Q)=\Delta_{1}+\Delta_{2}+\Delta_{3}
$$

où

$$
\left\{\begin{array}{l}
\Delta_{1}=D_{X}^{M-1}\left[W_{\alpha \beta}^{Q \cdot \tau}(X, t ; Q, \tau)\right]-D_{X}^{M-1}\left[W_{\alpha \beta}^{P \cdot \tau}(X, t ; Q, \tau)\right] \\
\Delta_{2}=D_{X}^{M-1}\left[W_{\alpha \beta}^{P \cdot \tau}(X, t ; Q, \tau)\right]-D_{X}^{M-1}\left[W_{\alpha \beta}^{P} \tau\left(X, t ; Q^{\prime}, \tau\right)\right] \\
\Delta_{3}=D_{X}^{M-1}\left[W_{\alpha \beta}^{P, \tau}\left(X, t ; Q^{\prime}, \tau\right)\right] \cdot[1-\gamma(Q)] .
\end{array}\right.
$$

En appliquant la limitation suivante, [2]

$$
\begin{gathered}
\left|D_{X}^{M-1} W_{\alpha, \beta}^{Q, \tau}(X, t ; Q, \tau)-D_{X}^{M-1} W_{\alpha \dot{\beta}}^{P, \tau}(X, t ; Q, \tau)\right|< \\
<\frac{\mathrm{const}}{\left(t-\left.\tau\right|^{\mu}\right.} \cdot \frac{1}{|X Q|^{n-1+M(1-q)}} \sup \left|A_{\alpha j}^{k_{1} \ldots k_{n}}(Q, \tau)-A_{\alpha j}^{k_{1} \ldots k_{n}}(P, \tau)\right|
\end{gathered}
$$


où $0<\mu<1$; les coefficients $A_{\alpha j}^{k_{i} \ldots k_{n}}$ sont hölderiens par rapport à la variable spatiale à l'exposant $h$ (hypothèse II), par conséquent

$$
\left|\Delta_{1}\right|<\frac{\text { const }}{(t-\tau)^{\mu}} \cdot \frac{\left.P Q\right|^{n}}{|X Q|^{n-1+M(1-\mu)}}, \quad \quad(0<\mu<1 \mid
$$

Étudions d'abord la différence $\Delta_{2}$

$$
\Delta_{2}=\frac{1}{(2 \pi)^{n}} \int_{-\infty}^{+\infty} \tau_{\alpha}^{\beta} \mid t, \tau ; P, \tau ; S,\left(i s_{1}\right)^{\gamma_{1}} \ldots\left(i s_{n}\right)^{\gamma_{n}}\left[\exp \left(i x_{n} s_{n}-i \sum_{\nu=1}^{n} s_{\nu} \xi_{\nu}\right)-\right.
$$

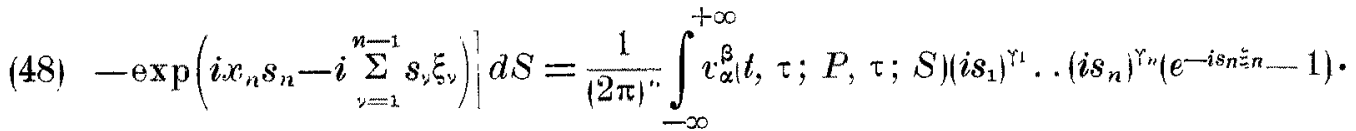

$$
\left.\cdot \exp \left[i 1-\xi_{1} s_{1}-\ldots-\xi_{n-1} s_{n-1}+x_{n} s_{n}\right)\right] d S
$$

où $\gamma_{1}, \gamma_{2}, \ldots, \gamma_{n}$ sont des nombres entiers non-négatifs, $\gamma_{1}+\ldots+\gamma_{n}=M-1$. Écrivons maintenanl cette différence sous la forme

$$
\begin{aligned}
& \Delta_{2}=\frac{(2 \pi)^{-n}}{(\sqrt[M]{t-\tau})^{n+M-1}} \int_{-\infty}^{+\infty} v_{\alpha}^{\beta}\left(t, \tau ; P, \tau ; \frac{z_{1}}{\sqrt{t-\tau}}, \ldots, \frac{z_{n}}{\sqrt{t-\tau}}\right)\left(i z_{1}\right)^{\gamma_{1}} \ldots\left(i z_{n}\right)^{\gamma_{n}} . \\
& \cdot\left[e^{-i \frac{z_{n} \xi_{n}}{M !}}-1\right] \exp \left[\frac{-i}{\frac{M}{V-\tau}-\tau}\left(\xi_{1} z_{2}+\ldots+\xi_{n-1} z_{n-1}-x_{n} z_{n}\right)\right] d z_{1} \ldots d z_{n}
\end{aligned}
$$

où $z_{k}=s_{k} \backslash \stackrel{\bar{t}}{t-\tau}, k=1,2, \ldots, n$. Les fonctions

$$
\begin{gathered}
v_{\alpha}^{\beta}\left(t, \tau ; P, \tau ; \frac{z_{1}}{\sqrt{t-\tau}}, \ldots, \frac{z_{n}}{\sqrt{t-\tau}}\right)\left(i z_{1}\right)^{\gamma_{1}} \ldots\left(i z_{n}\right)^{\gamma_{n}}\left|e^{-i \frac{z_{n} \xi_{n}}{M}}-1\right|= \\
=F_{\alpha}^{\beta}\left(t, \tau, P, \xi_{n} ; z_{1}, \ldots, z_{n}\right)
\end{gathered}
$$

sont des fonctions entières des arguments $z_{1}, \ldots, z_{n}$. En appliquant l'inégalité évidente

$$
\left|e^{-i \frac{z n \xi_{n}}{M}}-1\right|<\frac{\left|z_{n} \xi_{n}\right|}{\sqrt{M} t-\tau}
$$

et l'inégalité de Petrovsky, [2],

$$
\left|v_{\alpha}^{\beta}(t, \tau ; P, \tau: S)\right|<K e^{-\frac{1}{2} \delta s^{M}(t-\tau)}
$$


où désigne une constante positive, indépendante des paramètres $P$, $\tau$ et $s=\sqrt{s_{1}^{2}+\ldots+s_{n}^{2}}$; (o est la constante positive, fixée par l'hypothèse $\left.\mathrm{I}\right)$, nous aurons la limitation suivante

$$
\left|F_{a}^{\beta}\left(t, \tau, P, \xi_{n} ; z_{1}, \ldots, z_{n}\right)\right|<\text { const } \frac{\left|\xi_{n}\right|}{\sqrt[M]{t-\tau}} \exp \left(-\sum_{\nu=1}^{n} A_{\nu}\left|\tilde{z}_{v}\right|^{M}\right)
$$

où les constantes $A_{1}, A_{2}, \ldots, A_{n}$ sont positives; c. à. d. que les fonctions (50) sont de classe $Z_{M}^{M}$ au sens de GuELFAND et ChrLov, [4], par rapport à la variables $z_{1}, \ldots, z_{n}$. En vertu du théoréme de GuelFand-Chilov [4], nous en concluons que la différence (49) est de classe $Z_{q}^{q}$ par rapport à la variables $\frac{-\xi_{1}}{\sqrt[M]{t-\tau}}, \ldots, \frac{-\xi_{n-1}}{\sqrt[M]{t-\tau}}, \frac{x_{n}}{\sqrt[M]{t-\tau}}$, où $q=M / M-1$, donc

$$
\left|\Delta_{2}\right|<\frac{\text { const }}{(t-\tau)^{\frac{n+M}{M}}}\left|\xi_{n}\right| \exp \left[-\frac{C\left|X Q^{\prime}\right|^{q}}{\sqrt[M-1]{t-\tau}}\right] .
$$

En appliquant à la fonction (54) la méthode des séparations des singula. rités, présentée par W. Pogonzelski dans le travail [5] et en s'appuyant sur les inégalités

$$
\left|\xi_{n}\right|<\mathrm{const}|P Q|^{n_{L}+1}, \quad \chi \leq\left|\begin{array}{l}
X Q \mid \\
X Q^{\prime} \mid
\end{array}\right| \frac{1}{\chi}
$$

(ou $\chi$ est une constante positive inférieure à l'unité, [6]), nous arrivons à la limitation suivante

$$
\left|\Delta_{2}\right|<\frac{\text { const }}{(t-\tau)^{\mu}} \cdot \frac{|P Q|^{h_{L}}}{|X Q|^{n-1+M(1-\mu)}}, \quad \quad(0<\mu<1)
$$

$D^{\prime}$ après $(21),(43)$ et $(55)$ nous aurons maintenant

$$
\left|\Delta_{3}\right|<\left.\frac{\text { const }}{(t-\tau)^{\mu}} \cdot \frac{|P Q|^{2 h_{L}}}{\mid X Q}\right|^{-\mid m-1+\overline{M(1-\mu)}}, \quad(0<\mu<1) .
$$

Il en resulte, en vertu des inégalités (47), (56) et (57), que la différence (44) vêrifie l'inégalité

$$
\left|D_{X}^{M-1} W_{\alpha \dot{\beta}}^{Q, \tau}(X, t ; Q, \tau)-D_{X}^{M-1} W_{\alpha \beta}^{P, \tau}\left(X, t ; Q^{\prime}, \tau\right) \gamma(Q)\right|<\frac{\text { const }}{(t-\tau)^{\mu_{1}}} \cdot \frac{|P Q|^{h_{L}}}{\mid X Q^{n-1+M\left(1-\mu_{1}\right)}}
$$

où

$$
h_{L}^{\prime}=\min \left(h, h_{L}\right), \quad 1-\frac{h_{L}^{\prime}}{M}<\mu_{1}<1 .
$$


La différence (58) admet une limitation à faible singularité si $X=P$ et l'intégrale (42) tend vers une limite suivante

(60)

$$
\begin{gathered}
\lim _{X \rightarrow P} R^{(\alpha)}(X, t)=\lim _{X \rightarrow P} I_{2}^{(\alpha)}(X, t)= \\
=\int_{Q}^{t} \sum_{\beta=1}^{N} \varphi_{\beta}(P ; \tau) \iint_{S_{W}}\left\{D_{x_{1}}\left[D_{X}^{M-2} W_{\alpha \beta}^{Q . \tau}(P, t ; Q, \tau)\right]-\right. \\
\left.-D_{x_{1}}\left[D_{X}^{M-2} W_{\alpha \beta}^{P \cdot \tau}\left(P, t ; Q^{\prime}, \tau\right)\right] \gamma(Q)\right\} d Q d \tau= \\
=\int_{0}^{t} \sum_{\beta=1}^{N} \varphi_{\beta}(P, \tau) \iint_{S_{W}} D_{x_{1}}\left[D_{X}^{M-2} W_{\alpha \beta}^{Q, \tau}(P, t ; Q, \tau)\right] d Q d \tau .
\end{gathered}
$$

La première intégrale est absolument convergente, mais la seconde ne possède pas cette propriété.

D'après la limitation (58), l'intégrale de surface vérifie l'inégalité de la forme

$$
\begin{gathered}
\left|\iint_{S_{W}} D_{x_{2}}\left[D_{X}^{M-2} W_{\alpha \beta}^{Q . \tau}(P, t ; Q, \tau)\right] d Q\right|< \\
<\iint_{S_{W}}\left|D_{x_{1}}\left[D_{X}^{M-2} W_{\alpha \beta}^{Q, \tau}(P, t ; Q, \tau)\right]-D_{x_{1}}\left[D_{X}^{M-2} W_{\alpha \beta}^{P, \tau}\left(P, t ; Q^{\prime}, \tau\right)\right] \gamma(Q)\right| d Q< \\
<\frac{\text { const }}{(t-\tau)^{\mu_{1}}}
\end{gathered}
$$

à faible singularité, si $\tau \rightarrow t$; cette l'inégalité assure l'existence de l'inté. grale $(60)$, singulière relativement à la variable $\tau$.

Par conséquent, nous arrivons à la thèse (23) du théorème, c. q. f. d.

La valeur limite (23) de la derivée tangentielle peut être aussi exprimée par une valeur principale au sens de CAUCHr. Nous avons en effet

$$
\int_{0}^{t} \sum_{\beta=1}^{N} \varphi_{\beta}(P, \tau) \iint_{S_{W}^{\prime}-S_{\mathrm{II}}^{\prime}} D_{x_{1}}\left[D_{X}^{M-2} W_{\alpha \beta}^{P, \tau}\left(P, t ; Q^{\prime}, \tau\right)\right] d Q^{\prime} d \tau=0
$$

d'après la asymétrie centrale de la fonction

$$
D_{X}^{M-1} W_{\alpha \beta}^{P, \tau}\left(P, t ; Q^{\prime}, \tau\right)
$$

où $S_{\Pi}^{\prime}$ dẹsigne l'intersection par le plan tangent $x_{n}=0$ d'un cylindre II ayant pour l'axe $P x_{n}$ et de rayon $r_{\Pi}$, situé à l'intérieur du cylíndre $W$; done (64) $\lim _{X \rightarrow P} I_{0}^{(\alpha)}(X, t)=\lim _{r_{\square} \rightarrow 0} \int_{0}^{t} \sum_{\beta=1}^{N} \varphi_{\beta}(P, \tau) \iint_{S_{W}^{\prime}} D_{W_{n}[}\left[D_{X}^{M-2} W_{\alpha \beta}^{P, \tau}\left(P, t ; Q^{\prime}, \tau\right)\right] d Q^{\prime} d \tau=0$. 
Il en résulte que la valeur limite de la première partie de la dérivée tangentielle $(25)$ peut être exprimée sous la forme d'une intégrale singulière au sens de CAUCHY

$$
\begin{gathered}
\lim _{X \rightarrow P} D_{x_{1}}\left[D_{X}^{M-2} U_{\alpha}(X, t)\right]_{S_{W}}= \\
\left.=\lim _{r_{\Pi} \rightarrow 0} \int_{0}^{t} \int_{S_{W}-S_{\Pi}} \sum_{\beta=1}^{N} D_{x_{1}[} D_{X}^{M-2} W_{\alpha \dot{\beta}}^{Q} \tau(P, t ; Q, \tau)\right] \varphi_{\beta}(Q, \tau) d Q d \tau
\end{gathered}
$$

qui n'est pas absolnment convergente, où bien sous la forme d'une intégrale absolument convergente

$$
\begin{gathered}
\lim _{X \rightarrow P} D_{x_{1}}\left[D_{X}^{M-2} U_{\alpha}(X, t)\right] s_{W}= \\
=\int_{0}^{t} \int_{S_{W}} \int_{\beta=1}^{N}\left\{D_{\alpha_{1}}\left[D_{X}^{M-2} W_{\alpha \beta}^{Q, \tau}(P, t ; Q, \tau)\right] \xi_{\beta}(Q, \tau)-\right. \\
\left.-D_{x_{1}}\left[D_{X}^{M-2} W_{\alpha \beta}^{P, \tau}\left(P, t ; Q^{\prime}, \tau\right)\right] \gamma(Q) \varphi_{\beta}(P, \tau)\right\} d Q d \tau .
\end{gathered}
$$

Il en resulte aussi

$$
\lim _{X \rightarrow P} D_{x_{1}}\left[D_{X}^{M-2} V_{\alpha}(X, t)\right]=\lim _{r_{\Pi} \rightarrow 0} \int_{0}^{t} \iint_{S-S_{\Pi}} \sum_{\beta=1}^{N} D_{x_{1}}\left[D_{X}^{M-2} \Gamma_{\alpha \beta}(P, t ; Q, \tau)\right] \varphi \beta(Q, \tau) d Q d \tau
$$

c. à d. l'intégrale au sens de Cauchr.

Le théorème étudié dans ce travail m'a été suggéré par M. W. Pogo* RZELSKI; je tiens à lui exprimer mes sincères remerciements.

\section{OUVRAGES CITÉS}

[1] I. Perrovsky, Ueber das Cauchysche Problem für ein System linearer partieller Diffe. rentialgleichungen im Gebiete der nichtanalytischen Funktionen, "Bull. Univ. », Moscou, 7 (1938).

[2] W. Pogorzelskr, Étude de la matrice des solutions fondamentales au système parabolique d'équations aux dérivèes partielles, "Ricerche di Matem. " 7 (1958).

[3] W. Pogonzelskr, Propriétés des dérivées tangentielles d'une intégrale de l'équation parabolique, "Ricerche di Mat ", 6 (1957).

[4] Guelfand et Chrlop, Transformation de Fourier des fonctions de croissance vite (en russe), «Ouspieehi Matematicheskich naouk», Moscou (1958).

[5] W. Pogorzelski, Étude de la solution fondamentale de l' equation parabolique, "Ricerche di Matem. ^, 5 (1956).

[6] W. Poqonzersir, Propriétés des intégrales de l'équation parabolique, «Ann. Pol. Math. *, t. IV (1957). 